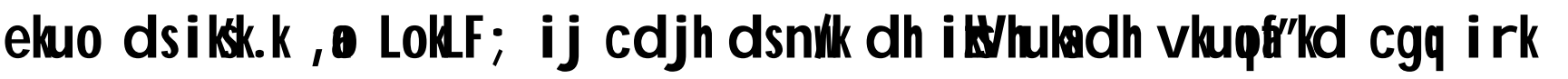 dkegio
}

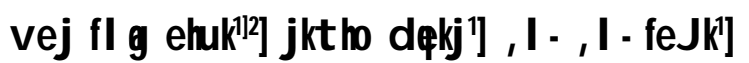

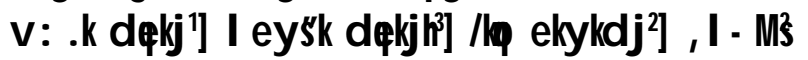

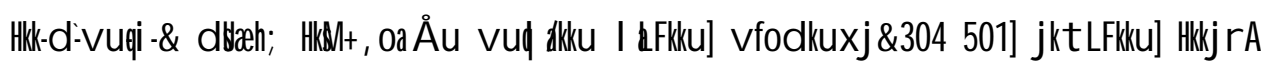

i $t$ tr\% $\%$ LL 2027

Lodèrof rej 2027

\section{I leflak}

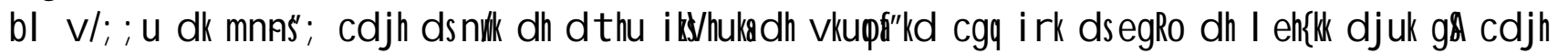

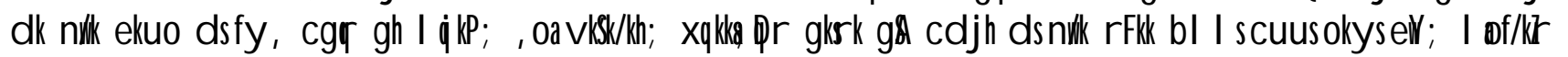

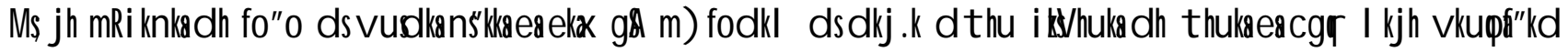

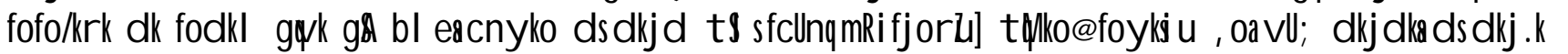

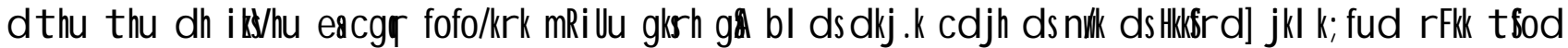

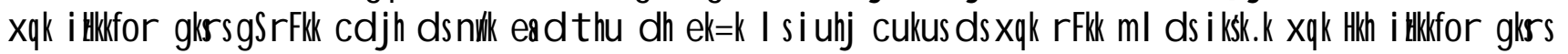

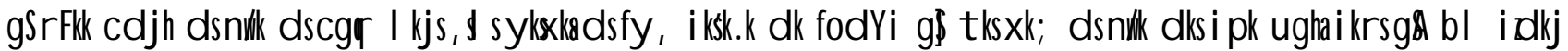

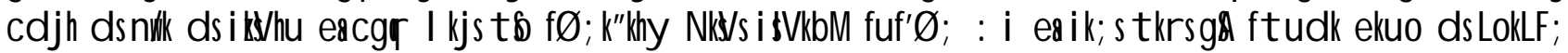

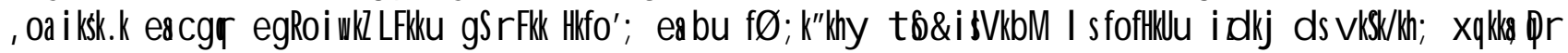

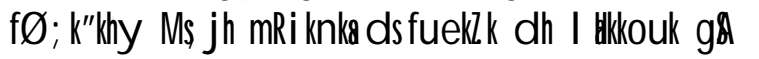

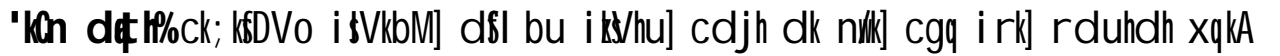

Bhartiya Krishi Anusandhan Patrika, 36(3): 173-177.

\section{Importance of Genetic Polymorphism of Goat Milk Proteins on Human Nutrition and Health: A Review}

\author{
A.S. Meena ${ }^{1,2}$, Rajiv Kumar ${ }^{1}$, S.S. Misra1, \\ Arun Kumar', Samlesh Kumari ${ }^{3}$, D. Malakar'², S. De ${ }^{2}$ \\ ICAR- Central Sheep and Wool Research Institute, Avikangar-304 501, Rajasthan, India.
}

Received: August 2021

Accepted: September 2021

\section{ABSTRACT}

The objective of the study is impotance of casein protein genetic polymorphism of goat milk. Goat milk is highly digestible and has medicinal properties in human being. Goat milk and its value added dairy products have higher demand in many contries of world. Due to evolution process, much genetic variability has been developed in casein protein genes. This is originatd due to point mutation; addition/ deletion and many other factors lead to huge genetic variation in goat casein genes. Because of this goat milk, chemical and biological properties have been affected. Goat milk casein contents decide

${ }^{*}$ Corresponding author's E-mail: amarsingh23@gmail.com

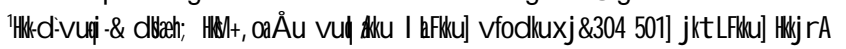
2jk'

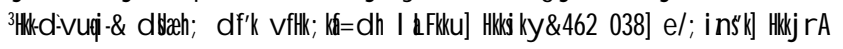

${ }^{1}$ ICAR- Central Sheep and Wool Research Institute, Avikangar-304 501, Rajasthan, India.

${ }^{2}$ National Dairy Research Institute, Karnal-132 001, Haryana, India. ${ }^{3}$ ICAR- Central Institute of Agricultural Engineering, Bhopal-462 038, Madhya Pradesh, India. 
the cheese making properties as well as nutritional and medicinal values. Goat milk is good alternative source of nutrition in human when people have cow milk problems. Goat milk has humerous inactive bioactive peptides in their casein protein. These have important place for human health and nutrition. In the future, this is great possibility for development of medicinal value functional dairy products from functional bioactive peptides.

Key words: Bioactive peptide, Casein protein, Goat milk, Polymorphism, Technological properties.

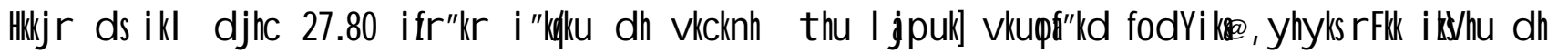

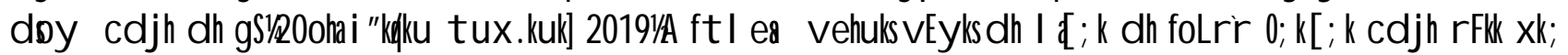

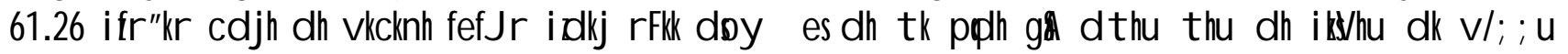

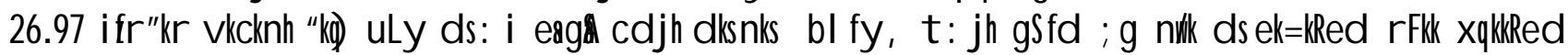

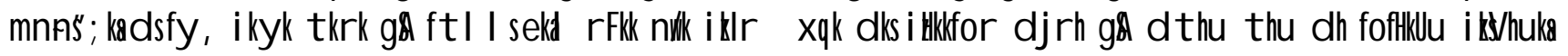

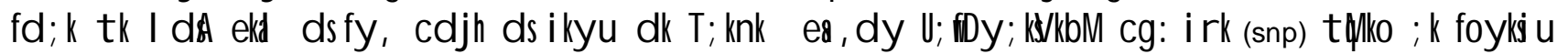

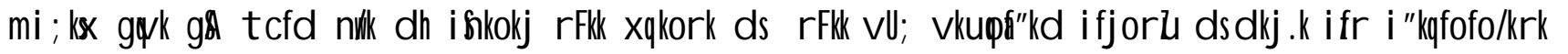

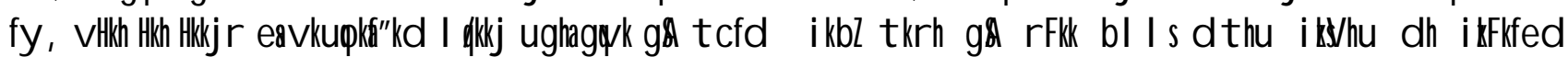

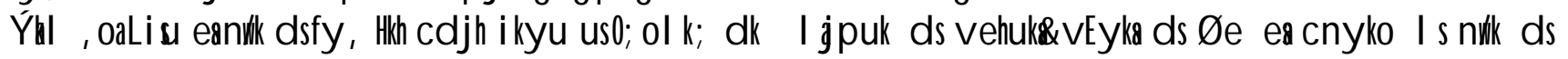

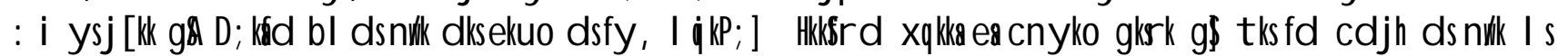

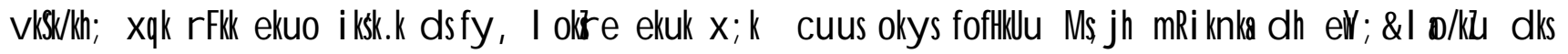

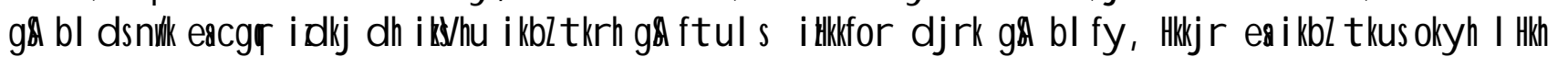
ekuo dsi kkkdsfy, coq I sevy I efl

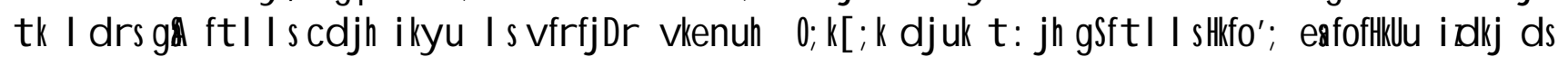

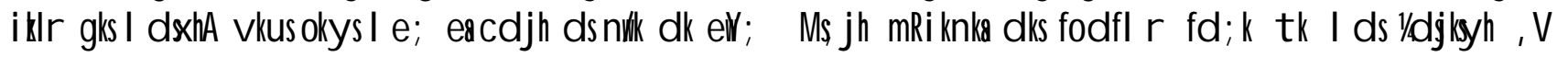

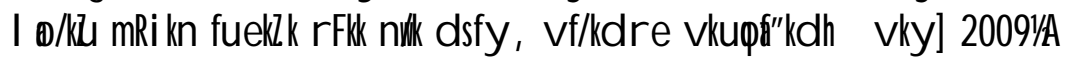

p; u I scdjhi kyd dhvlenuhc łused gk d fl ) glska bl dsfy, ds he i tster dsfoflteu vkuqäld

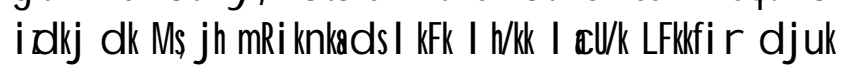
gls|Aft I I sh dsmiR knledkolft c nle fey Acdj hds

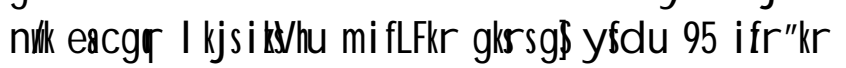

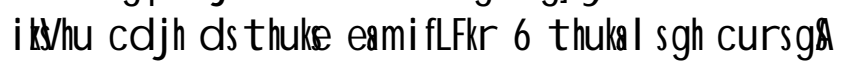

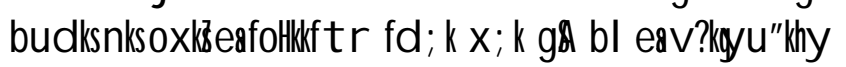

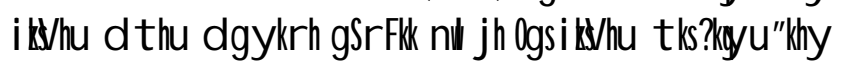
i tste gaft I espli dt he t he i zyrkl s, d nijs s

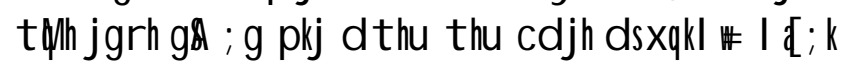
6 ij 250 fdylst dsthy ij miflhr glshg\&t l ea

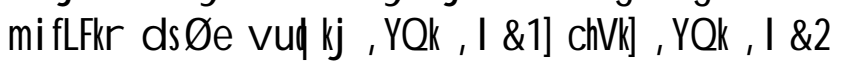

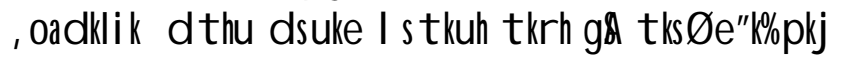

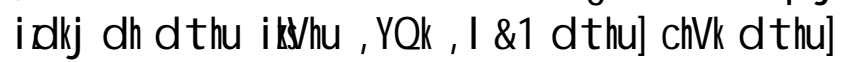
, YQk, I \&2 dt hu, oadki kdt tu i tste ds. i eank

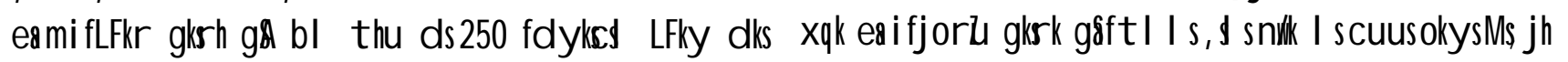

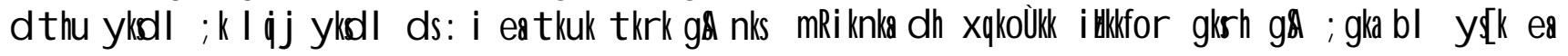
Qsi

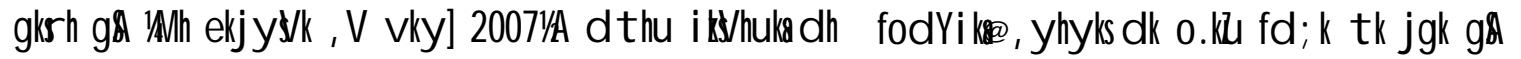




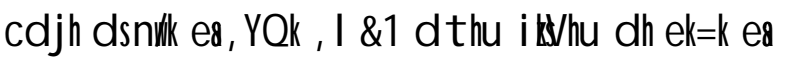
I cl sT; knkvidj fofttul ulyleds nL; feyrkgd vHthrd fo"o ea23 vkuqf'ld fodYi [ Hos st kpdsga mijlit vkuqa'ld fodY ledhfdl hi "kepaniflik I s

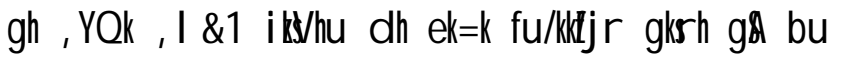

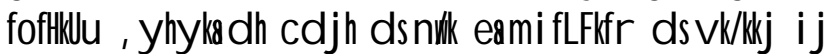
pli ox cakx; kgSi3, yly dlsizy , yly ds. i eet kukt kkg\$D Wudhcdj heamiflifir I sgh , YQk, I \& I dt hu i tste dhekk kvf/ld j grhgsddi to

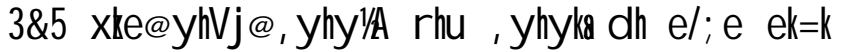
128_d\&]\&8 xle@y

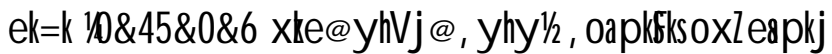
, 5s, yly thi[ Hos st kpdsgoft udhcdjhdst tole ea miflHfir I s, YQk, I \& dt tu d hnvikeakkdkvHto ; kvuiofliffr jgrho\$budlsuy, yly dgr soA bl i d d k , YQk, I \&ldt to i tsto dhnip ; kde ekkdh

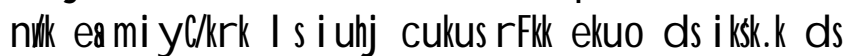
m; is dscog I ksevy I offle miR kn cukusdsfy, m; kx esfy; kt kl drkgSXhelkyłk, V vły] 2007 , oadckg, V vky] 2016^A

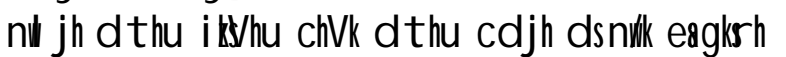
gSft I dhekk viv dt to it:ter I svf/ld glsh gS ft I dsvithrd 9, yly cdj hdst tule esi rkfd; st $k$

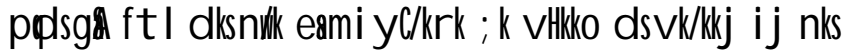

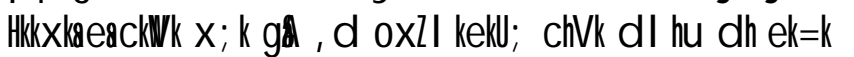
nuk eacuk sj [ kk gSr Fk nhy soxZdsl nL; eachk

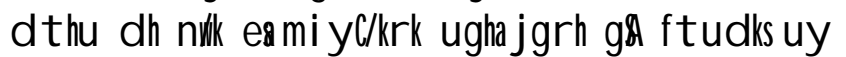

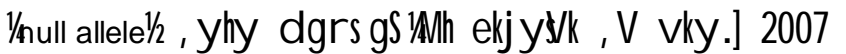
, oadlls $\mathrm{H}$ k, V vky.] 20121 A

rth j s t:tu, YQk, I \&2 dt thi glsko\$ft I dsvith

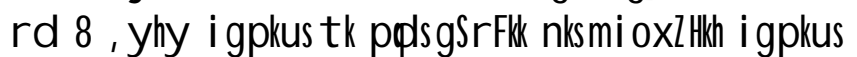

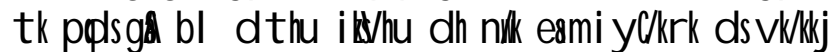
ij rlou Hexloeackkt kl drk g\$ i lp ,yly , dy

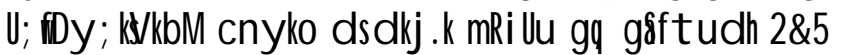

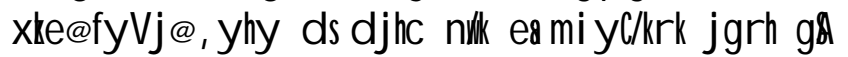
rhknk, yly dhekk nukeacga ghde jgrhgs Hk uy , yly dsalk.kbl i tstu dhek kdknolkear/tko glsk \$\$t lsfd , dy feluonti fj or Z dsdlk. ki tstou

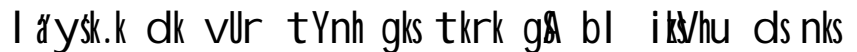
moxZthi kst ksg\$t kfd I etak , yly ds4 vflue vehulsvE/ dkfoylisu I tofdulksdhvlis glst kkgS 3Xheljy \&k, V vly] 2007, od \$olkh, V vly] 2014A

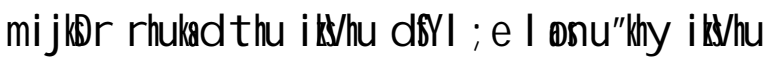

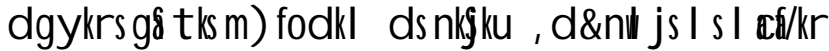

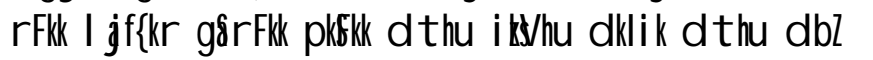

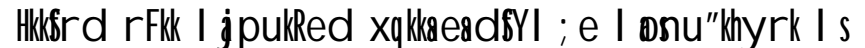

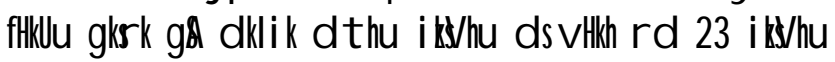
, yly i rkglspdsgat udlsvibl lsby vklk ij nlsl egy eafoltht $r$ fd; kx; k gA cld egy , yly eadlij kdt hi dhekk, \& eg/dhvi \&kndkea

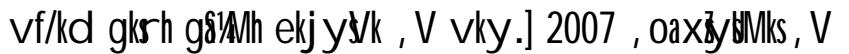
vly.] 2015A

mjj lis pliks tstu dhekkdlsnykeavf/ld djus

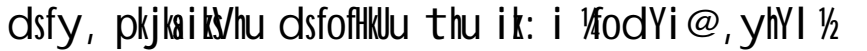

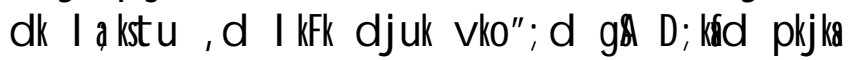
dt hu i tste dsfoftted, ylyledk, d \&nly sdt ter dh

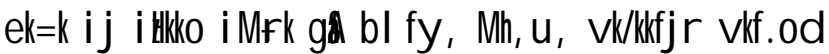

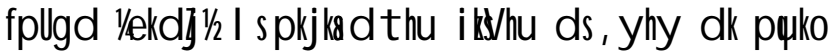
djuk vio"; d g\& Hikr, df'k vulatke i fj'kn] ubZ fnYyhdsdjuly] ] eflikk, oavfodkuxj dsoßkłud lsdh

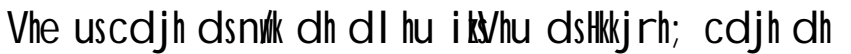
uly la ks kusolys svkuqäld cg: i r kdki r kyxk k

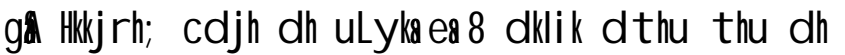

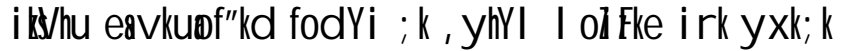

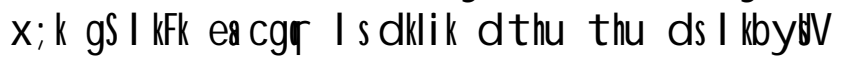
of, W , oangl yH dk thi rkHtirh, cdjhdh

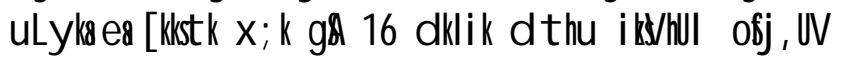

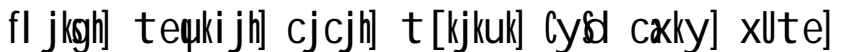
j $k x<$, ofef $r$ cdj hdsulyladst kuoj les, D $k$ \& 4 i j i k sx; sgStxlse , V vły] 2019 A bl I s; g fl )

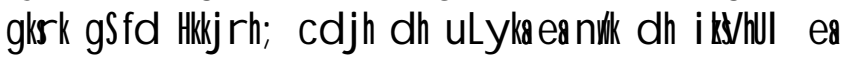
cga vkuqaild fofo/kk i kbz kh ga in vkuqa'ld fofo/kkdlscdj hdsnikl scuusoly si\$j hntR knleds

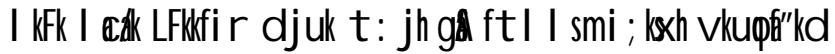
o\$, W dsfy, cdj hdst kuoj ledkp; u fd; kt kl dA

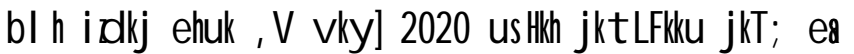
i koz kusoly hfl j lshcdj ht [ likkukcdj h, oadjolkh cdj hesth, YQk, I \&1 dt he] , YQk, I \&2 dt hu , Oa

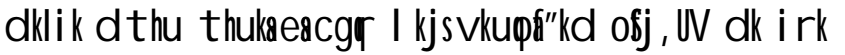
yxk k x; k gd bl eacdjh ndk dsl astolgd ,e\& vki, $u$, dhvure dhHhQlk; kdhxbzgsxuli fyl M

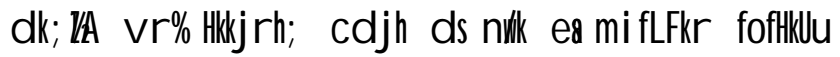

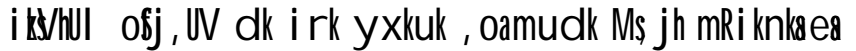
m; r khi Hto dst eakdlst kuukt : jhgAft I I scdj h 


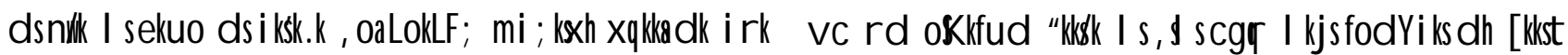
$\mathrm{fd} ; \mathrm{kt} \mathrm{kl} \mathrm{dA}$

\section{cojjhdholt hu t hu cgai rkdki Lbo, cagpo}

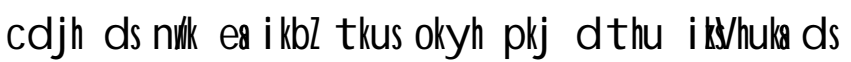

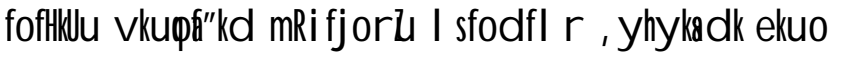

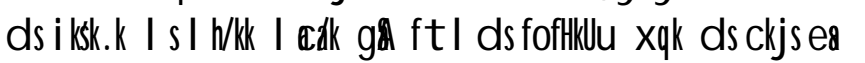
folr li I so. la bl yskeefd; $k x ; k g$

\section{'d1/Rduld xok}

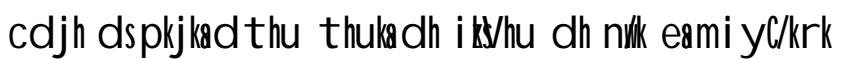

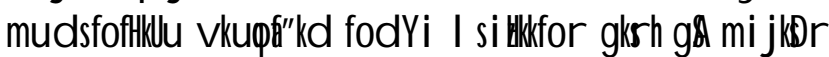

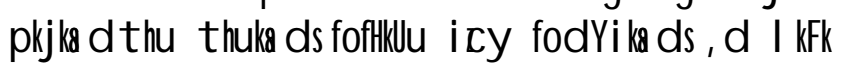

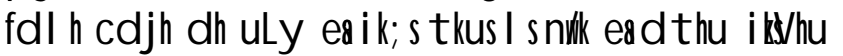

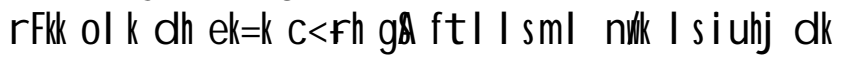

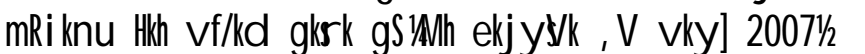
rFlkbl hi dlik noz fodYl ledhfdl hcdjhdsuly ds

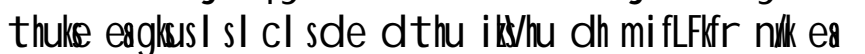
glsi kn gSt I I smh nuk I si ulj dknkrku ux.;

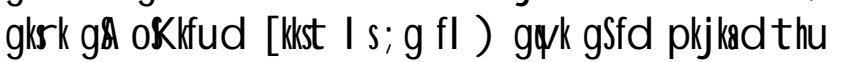
dsfofthlu fodY leds als u I s, sst hui $\mathrm{k}$ i dkpqlo fd; kt kl drkgAft I dsdlk.kcdjhdsnykl si ubj

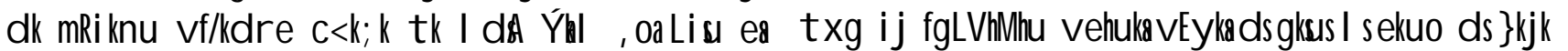

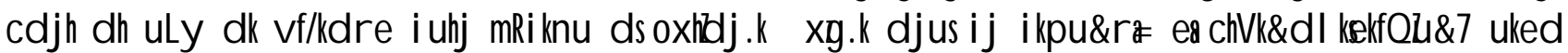

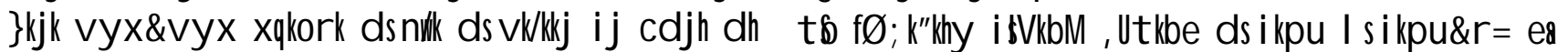

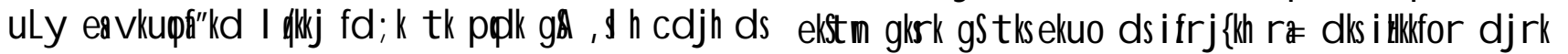
Msj hnir knled he nir knu I sT; knkgSXhelky $\$ k$

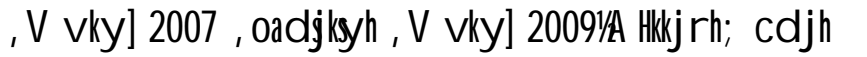

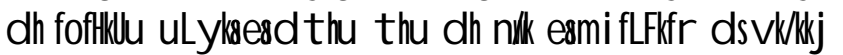
i j i gplu djukt : jhgst I I sfd i ulj miRku ds fy, m; q i "kqledkpqlo fd; kt kl dsr HkHkir ea

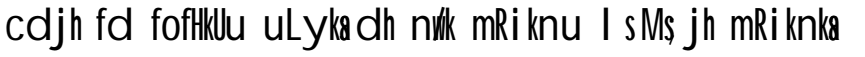
dk fuekk fd; kt k I dA bl ds\}lkk cdj hi kyu I s vfrfi $\mathrm{D}$ ylktkdek kt kl dz|A

\section{Ifak zyt la scplo}

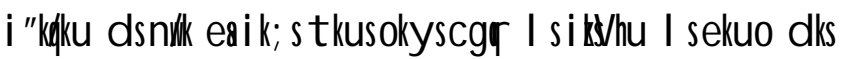

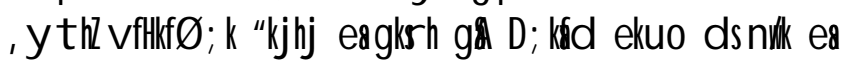

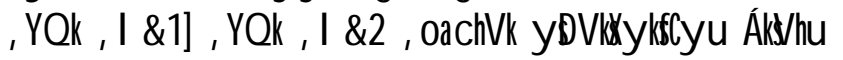

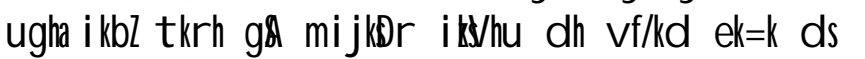

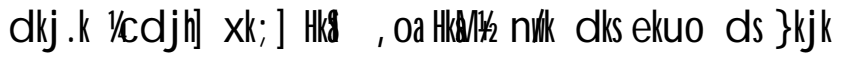
xg.kdjusi j "kith ea yt buffffo; kglshg\$t lsfd fcukl ko/kuhdsetuo dsfy, udl kunk d gabl fy,

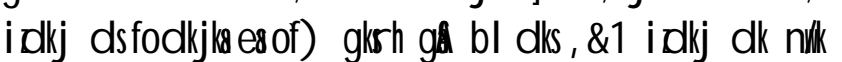

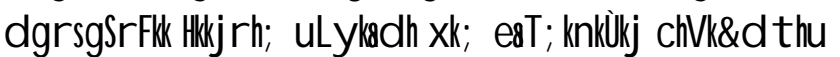

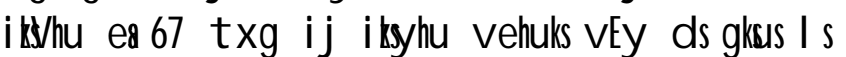

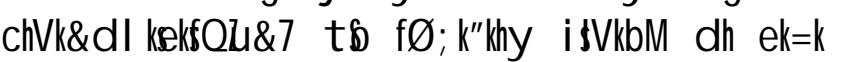
vkp; Zud : i I sole glst khofabl dls, \&2 feYd dgr sge is, \&l feYd dsglfudlixd i tto dlsole dj rk gStdj h, V vk ] 2009A vf/ld ekkea \&l feYd dk m; ix djusl sekuo dsLoltF, ij glfudlid i tto

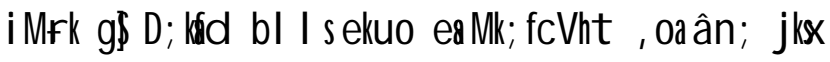
glksisdhl Houk c<tt khgd bl fy, chM\&dt to ea

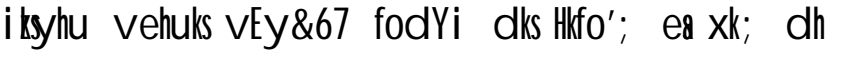

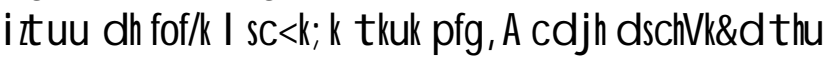

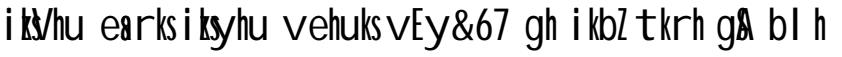

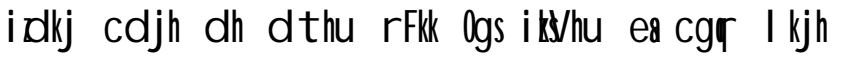

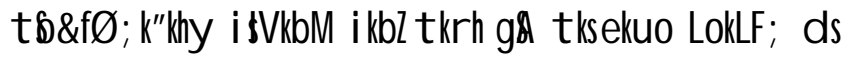




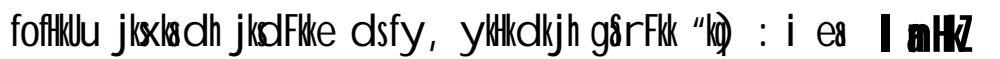

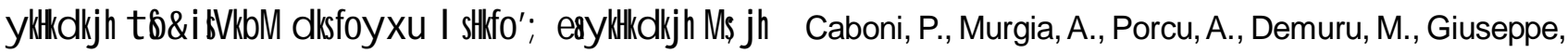
nRR knladkfuelkfd; kt kl drkg\$cdj hdswkdkekio

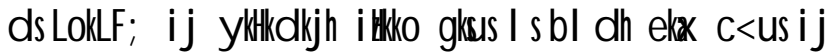

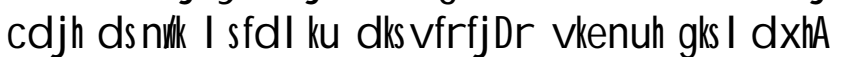

\section{fưd'l'z}

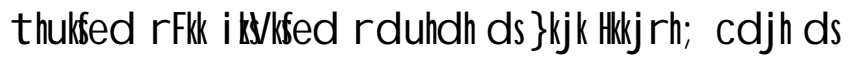

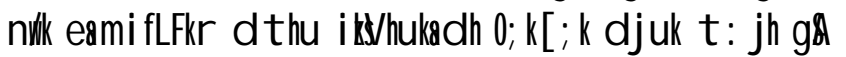

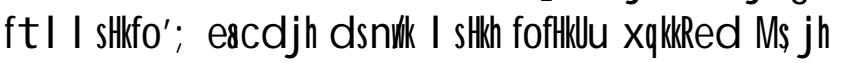

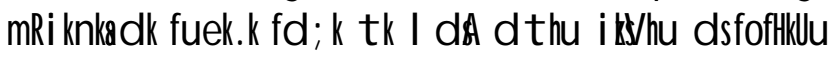
vkuç'ld fodY ledsp; u I s uln nRR knu] ndile ekuo

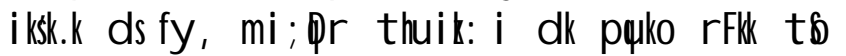
fO; K'Ky i BMbMdhi golku I stfo'; efoftkel i dlik ds

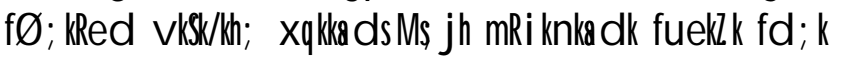
t kl drk gA bl I scdj hdsn/k dkvf/ldre m; k fd; kt kl ds|A nuk dsi tshe dhvikuga'ld fofo/kk

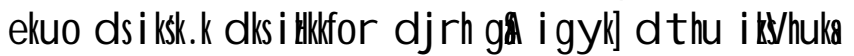

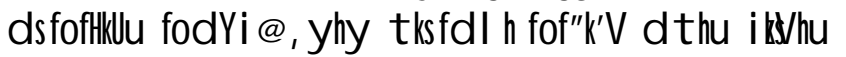
dhekkd lsnkesde dj rkgsinh d lsekuo ds lskkds

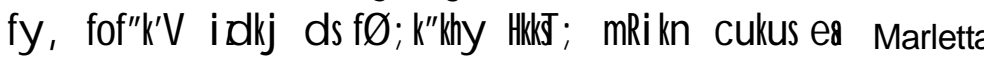
m; k esfy; kt kl drkgAt Ssde, yt bolykndAA bl hi dlik vif. od fpled dsl g; la I scdjhdsi z uu

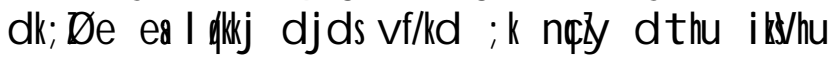
fodY dkpqlo fd; kt kl drkga Hfo'; excdjhds

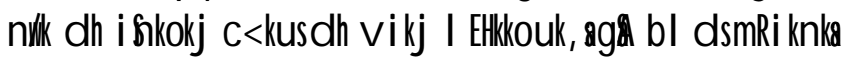
dhelx i gys sghi slokj dhvi \$kT; knkg\$bl dsnke

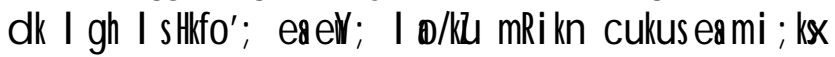
glssis j fall ku dhvlenuhc «tused gk d fl ) gks|A
P., Nudda, A. (2016). Gas chromatographymass spectrometry metabolomics of goat milk with different polymorphism at the alpha S1 casein genotype locus. J. Dairy Sci. 99: 1-6.

Caroli, A., Chessa, S., Erhardt, G. (2009). Milk protein polymorphism in cattle: Effect on animal breeding and human nutrition. J. Dairy Sci. 92: 53355352.

Cosenza, G., Lannaccone, M., Pico, B.A., Ramunno, L., Capparelli, R. (2012). The SNP g1311T>C associated with the absence of beta casein in goat milk incluences CSN2 promoter activity. Animal Genetics, doi10.1111/age.12443.

Gautam, D., Vats, A., Verma, M., Rout, P.K., Meena, A.S., Ali, M., Deepika, S., De, S. (2019). Genetic variation in CSN3 exon-4 region of Indian goats and a new nomenclature of CSN3 variants. Animal Genetics. 50: 191-192.

Gerlando, R., Tortorici, L., Sardina, M.T., Monteleone, G., Mastrangelo, S., Portolano, B. (2015). Molecular characterization of kappa casein gene in Girgentana dairy goat breed and identification of two new alleles. Italian Journal of Animal Science. 14: 90-93.

, D., Criscione, A., Bordonaro, S., Guastella, A.M. and D'Urso, G. (2007). Casein polymorphism in goat's milk. Lait. 86: 491-504.

Meena, A.S. (2020). Molecular characterization of casein gene(s) in Sirohi goat (Unpublished Ph. D thesis). ICAR-National Dairy Research Institute, Karnal132001 (Haryana), India.

Selvaggi, M., Laudadio, V., Dario, C., Tufarelli, V. (2014). Major proteins in goat milk: An updated overview on genetic variability. Mol. Biol. Rep. 41: 10351048. 